\title{
NIRSpec - an IR spectrometer for the James Webb Space Telescope $t$
}

Wolfgang Holota, Holota Optics, Auguste-Wittig-Str. 42, 83646 Bad Tölz, Germany

Maurice B.J. te Plate, European Space Agency, Keplerlaan 1, PO Box 299, 2200 AG, Noordwijk, The Netherlands

Abstract:

NIRSpec - an IR spectrometer for the James Webb Space Telescope ${ }^{1,2,3}$

The near-infrared spectrograph (NIRSpec) is part of the James Webb Space Telescope (JWST) science mission.

The JWST Observatory, a cooperation of NASA and ESA, the follow-on mission to the Hubble Space Telescope and to the Spitzer Space Facility, will yield astounding breakthroughs in the realms of infrared space science.

$\mathrm{NIRSpec}$ is a spectrograph that works in the near infrared spectral region from $0.6 \mu \mathrm{m}$ to $5 \mu \mathrm{m}$ and allows the observation of spectral features of the incident light with different spectral resolutions $(R=100$, $R=1000, R=3000$ ). It is designed for spectroscopy of more than 100 objects simultaneously. The optical design of the NIRSpec instrument is characterized by a straight optical system layout: It constitutes of a set of optical modules of similar optical design type with high performance and low module tolerances. The NIRSpec instrument development is lead by the European Space Agency with EADS Astrium GmbH (Germany) as prime contractor for instrument development, design, and manufacturing.

Keywords: Deep Space, Spectrometer, Infrared, Deep Temperature, IR Large Telescope

\section{INTRODUCTION}

The JWST science mission, a cooperation of NASA and ESA, intends to observe a variety of deep space scenarios, especially focused on the light-waves that have been stretched - and red-shifted - by the expansion of space since the Big Bang.

The key astronomical research tasks are the identification of the first light after reionization, the understanding the assembly of galaxies, the formation of stars and planetary systems and the evolution of planetary systems and the origins of life.

In order to achieve the above listed science goals, the JWST mission comprises an optical telescope (OTE) with a large segmented primary mirror (6,6 m diameter) and a set of three scientific instruments: NIRCam (Near-Infrared Camera), NIRSpec (Near-Infrared Spectrograph), and MIRI (Mid-Infrared Instrument). NIRCam is a refractive, imaging optical instrument that works in the visible/near-infrared spectral region $(0.6 \mu \mathrm{m}-5 \mu \mathrm{m})$. NIRSpec represents the mission's spectrograph for the visible/nearinfrared region. It provides several modes for spectral analysis of the star light with low, mid, and high spectral resolution. MIRI is an imager /spectrometer/coronograph in a spectral range of $5.0 \mu \mathrm{m}$ to $28 \mu \mathrm{m}$. In addition, a fine guidance sensor (FGS) tunable filter and guider is installed on JWST in order to provide highly accurate pointing knowledge. The FGS tunable filter also provides science capabilities. orbit.

The JWST telescope is passively cooled to $(30-50) \mathrm{K}$ at the thermally stabile L2 LaGrange point

The input light of these instruments is provided by the James Webb Optical Telescope (OTE) which is based on a Korsch-type optical design. Moreover, its image is not plane but on a spherical surface (spherical field curvature) and a wave-front error of less than $145 \mathrm{~nm}$ shall be achieved throughout the whole field of view for the real system.

In this article the focus is on the scientific tasks, the optical design and lay-out and the first test results of NIRSpec. 


\section{CHALLENGING REQUIREMENTS}

Some aspects of the instrument requirements are challenging the development and the manufacturing of the NIRSpec instrument optics.

\subsection{NIRSpec to OTE Optical Interfaces}

There are two important optical interfaces between the optical telescope (OTE) and the NIRSpec scientific instrument. First, the OTE primary mirror edge represents the optical system aperture stop and, thus, limits the NIRSpec light beams.

Secondly, the OTE provides the NIRSpec object field, which is not a plane but an off-axis section of a sphere with a radius of $3017 \mathrm{~mm}$. For this reason the NIRSpec optical design has a field of view orientation which is symmetric with respect to the polar OTE geometry. This allows a compensation of the field curvature by the NIRSpec optical mirror design. Additionally, tolerances of the OTE focal surface position due to OTE manufacturing, OTE alignment and due to changes at operational conditions are expected to be of the order of $+/-3 \mathrm{~mm}$. Therefore, NIRSpec provides a refocusing mechanism that adapts accurately to the correct OTE focal surface position.

\subsection{Spectrometer Slits for Multi-object Spectroscopy}

The spectrometer slits cannot be positioned at the NIRSpec object surface since this surface is not plane and since a spectral filtering is necessary before the light enters the spectrometer (see NIRSpec observation modes). Additionally the current image-scale at the OTE focus needs to be increased to fulfil the required minimum Field of View requirement at the plane of the slits. Therefore, the slits are positioned at an intermediate field plane.

Multi-object spectroscopy of more than 100 star objects is achieved using a so-called MEMS system (Micro Electro Mechanical System), which comprises an array of more than 250.000 small mechanical shutters (micro-shutter array, MSA) each equipped with a small "door" of a size of 80 micron times 180 microns (spectral dimension times spatial dimension) which can be open or closed. Those shutters that belong to the objects of interest are opened and the spectra measured. Note that a complete row of shutters becomes useless if a single shutter stays open, since the spectrum of the light of the open shutter can cover up to a full detector row. The manufacturing of this sophisticated micro-shutter subsystem is under responsibility of NASA.

For high spectral resolution there are some additional slits with exact edges at fixed positions at the intermediate field plane. The object of interest is positioned at the centre of one of these fixed slits (FXSL).

Moreover, a complete small star field region of 3arcsec times 3arcsec can be analysed spectrally via a splitting of the star region image into small stripes. Each of these stripes represents a spectrometer slit and allows a spectral investigation. The unit that provides the field slicing and enables the spectral analysis of such a star region is called the integral field unit (IFU).

\subsection{Target Acquisition}

It is intended to position the scientific objects very precisely within the apertures of the micro-shutters or fixed slits. This is achieved by using the so-called target acquisition procedure. During this target acquisition procedure, the sky field is correctly mapped onto the grid of MSA shutters using a combination of OTE small angular manoeuvres and the OTE's fine steering mirror mechanism. The acquisition of the scientific target with integration times of the order of 1000 seconds requires a very stable imaging optics: Position stabilities that correspond to a few milli-arc-seconds (mas) at sky are necessary.

\section{NIRSPEC OBSERVATION MODES}

NIRSpec provides several modes for direct imaging and spectral analysis of star objects. First, in the Imaging mode NIRSpec allows a direct observation of the star configurations with the capability to block unwanted bright objects in the scene. Furthermore, a dedicated target acquisition mode guarantees the precise positioning of multiple star objects on a versatile slit configuration of the MSA. In imaging mode the so-called target acquisition mirror or TAM is selected at the grating wheel mechanism (GWA). 
Secondly, a low spectral resolution mode, the $R 100$ mode, gives a spectral overview within the full spectral range between 0.6 micron and 5.0 micron. The dispersing element is a prism, and the spectrograph design is such that an average spectral resolution of about $R=100$ is achieved in this mode.

Third, NIRSpec provides a spectral mode with medium spectral resolution of about $R=1000$, the $R 1000$ mode. In this mode a grating is used as dispersing element. In order to avoid an overlap of the spectrum of interest with higher diffraction order spectra and to optimize instrument throughput, the spectral range is split into three sub-ranges $(1.0 \mu \mathrm{m}-1.8 \mu \mathrm{m}, 1.7 \mu \mathrm{m}-3.0 \mu \mathrm{m}$, and $2.9 \mu \mathrm{m}-5.0 \mu \mathrm{m})$. For each band a dedicated grating is available at the GWA.

Fourth, a high spectral resolution mode, the $R 3000$ mode, with a spectral resolution of $\mathrm{R}=3000$ is available. As for the medium spectral resolution mode the spectral range is divided up into three spectral bands, and three different gratings are used for resolving the spectra. As in the R1000 mode, a set of three spectral filters avoids that the spectra of different diffraction orders overlap at the detector.

Finally, in the Calibration mode a spectral and a distortion calibration are performed in order to determine correctly the precise spectral information and to support the highly accurate target acquisition.

\section{NIRSPEC OVERALL DESIGN}

The NIRSpec optical train consists of two main subsystems: The so-called fore optics (FORE) and the spectrometer optics (SPEC).

The FORE optics is an optical imaging system that maps the OTE image sphere section onto the intermediate field plane, where the micro-shutter array is located. It consists of three aspherical mirrors responsible for the imaging and a set of plane mirrors for beam redirection. The FORE optics design must cope with the curved object surface, a well corrected pupil plane, a telecentric image, and an excellent optical performance.

The second part of the NIRSpec optical train is the spectrometer-optics (SPEC) which picks up the light at the intermediate image plane, guides it to the dispersing elements, and images the spectra onto the sensitive area of a detector.

In order to create the desired slit pattern, multiple micro-shutters can be opened at the same time. As the spectrometer optics is telecentric at its object side the geometric loss of light at the open microshutters is minimized and the symmetry within the MSA plane is conserved.

The spectrometer consists of a single optical system, the spectrometer-optics, working together with one of the seven dispersing element (the prism, or one of the 6 different gratings) allowing spectroscopy with three different spectral resolutions. The spectrometer optics can be split into a first optical module, the collimator optics (COL), that collimates the rays of any field position, and a second optical module, the camera optics (CAM), that images the dispersed light rays onto a detector.

In Fig. 1 the optical design of the NIRSpec instrument in the imaging mode is shown. 


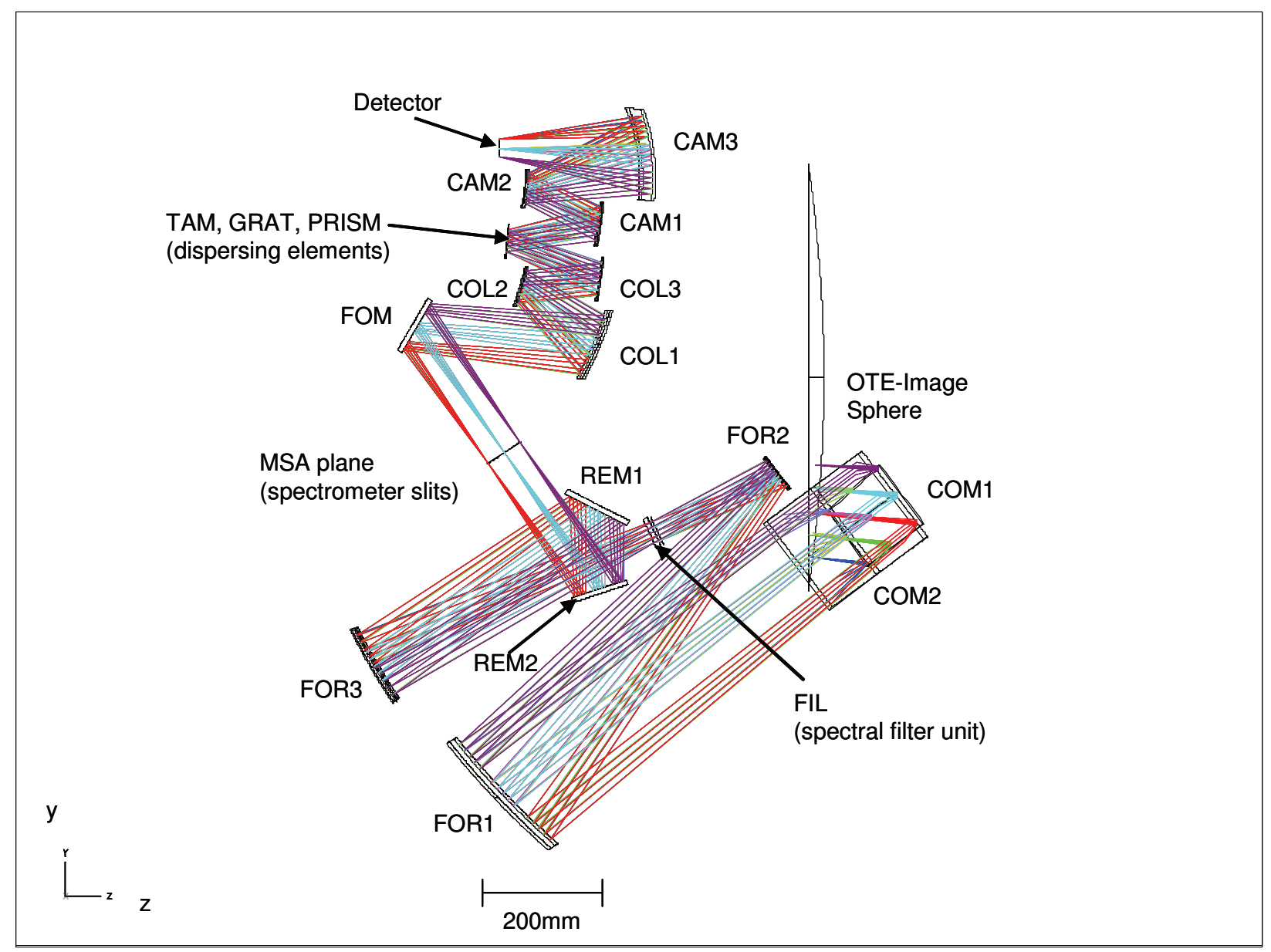

Figure 1: Optical Design of the near-infrared spectrograph (NIRSpec): The FORE-optics images the spherical object surface section onto the intermediate image plane; the spectrometer optics (SPEC) in the imaging mode maps this intermediate image onto the detector plane. At the intermediate image the variable multi-spectrometer-slit assembly is positioned, whereas the detector position is at the spectrometer optics image. The spectral filter unit is located at the FORE-optics pupil plane; the dispersing element unit is positioned at the spectrometer optics pupil plane.

\section{THE FIRST TEST RESULTS}

NIRSpec recently finished the first part of its calibration campaign. In this so-called CRYO1 phase, MSA usage was restricted due to an issue which is currently under investigation by NASA. As a result, only fixed slit spectra and IFU spectra could be collected. Besides this, spectra were collected through the "failed open" MSA shutters.

Despite this significant operational restriction, a lot of performance information was obtained during this test campaign. Preliminary analysis of the calibration data shows that the optical mechanical aspects of NIRSpec are in an excellent shape. The optical alignment turns out to be very good, all dispersive elements behave according to expectation, fixed slits and IFU perform as intended. Furthermore, all mechanisms are working very well and there are no signs of molecular contamination.

As an example, we show a NIRSpec spectral image that has been obtained using the external Argon lamp source that is part of the NIRSpec optical test equipment. 


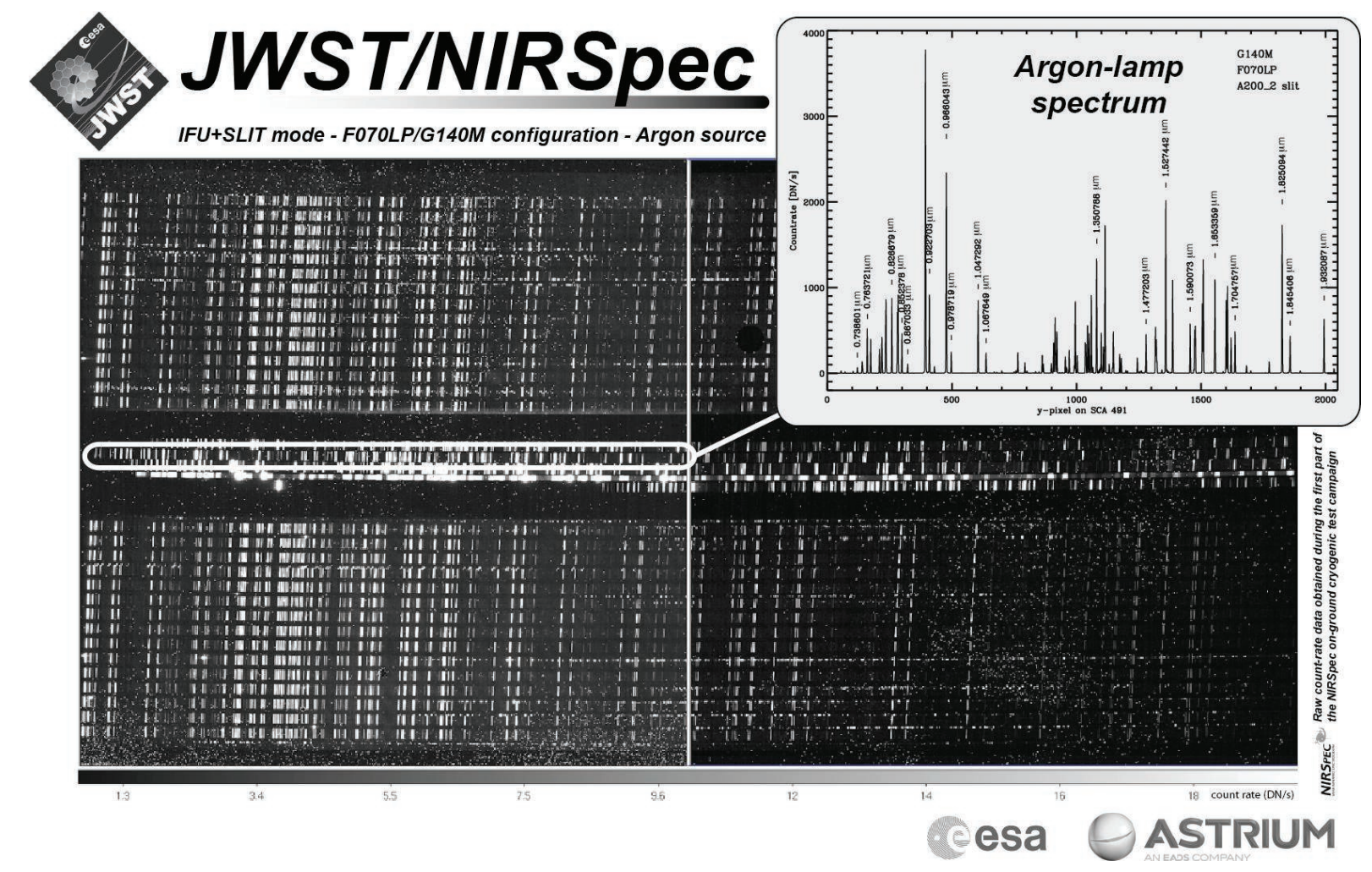

Figure 2: NIRSpec fixed slit and IFU Argon lamp spectrum collected with the $\mathrm{R}=1000$ grating in spectral band $\mathrm{I}$.

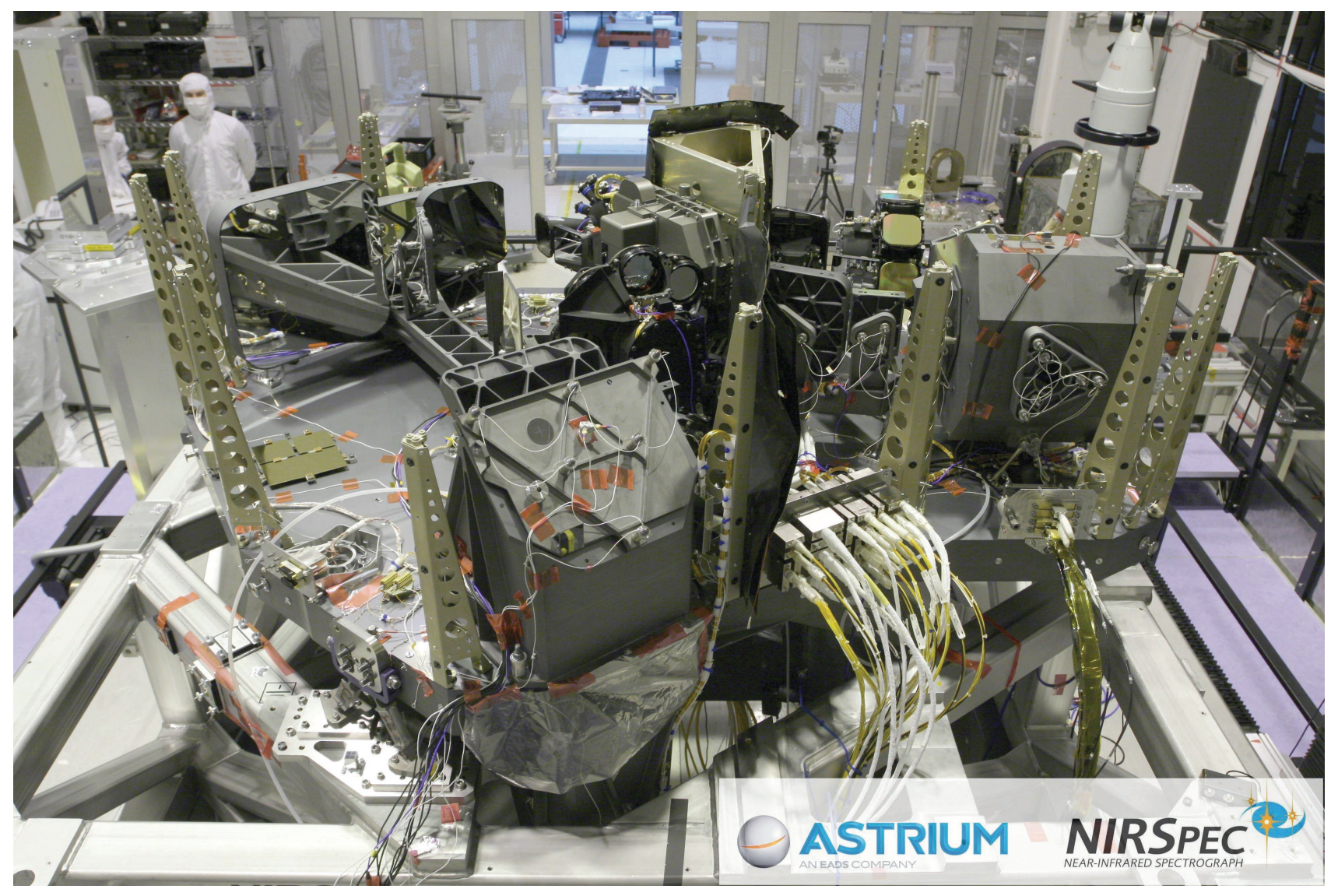

Figure 3: The flight model of NIRSpec at Astrium GmbH 


\section{SUMMARY}

With this article we have explained the essential parts of the optical design of the NIRSpec instrument, which is part of the JWST science mission. The interaction between important required features and the optical design aspects was shown to have lead to an optical system design with several modules: The FORE optics maps the spherical NIRSpec object field onto an intermediate field plane where the multi-object variable spectrometer slit arrangement is positioned. The imaging performance at this intermediate plane is very good. At the well corrected pupil plane of this first optical imaging system the spectral filter assembly is located. The SPEC optics maps the plane, intermediate field onto the sensitive detector area with smaller dimensions. In addition to this imaging mode, which is used for target acquisition, a spectrometer with variable spectral resolution is realized if the mirror at the spectrometer optics pupil plane is replaced by a dispersing element. A mechanism allows the selection of the imaging mode mirror, a prism for low spectral resolution (R100 mode) and six different gratings for medium (R1000 mode) and high spectral resolution (R3000 mode). The spectrometer-optics modules are a collimator (COL) and a camera optics (CAM). The star object light of interest is collimated by the COL module, the dispersing elements transform the spectral information into exact ray directions, and the CAM optics guides these rays to definite positions at the detector, thus, forming accurate spectra. The design optical performance at the image surface is shown to be very good for all operational modes.

\section{REFERENCES}

${ }^{1}$ J. Koehler, M. Melf, W. Posselt, W. Holota, M. te Plate: „Optical Design of the Near-infrared Spectrograph NIRSpec“ ,Proc.SPIE Vol. 5962,5961V, 2005.

${ }^{2}$ P. Davila, B. Bos, J. Contreras, C. Evans, M. Greenhouse, G. Hobbs, W. Holota, L.W. Huff, J. Hutchings, T.H. Jamieson, P. Lightsey, C. Morbey, R. Murowinski, M. Rieke, N. Rowlands, B. Steakley, M. Wells, M. te Plate, G. Wright: "The James Webb Space Telescope Science Instrument Suite: An Overview of Optical Designs", Proc.SPIE Vol.5487,611, 2004

${ }^{3}$ M. te Plate, W. Holota, W. Posselt, J. Koehler, M. Melf, G. Bagnasco, P. Marenaci: „Opto-mechanical design of the Near Infrared Spectrograph NIRSpec", Proc.SPIE Vol.5904,59040L, 2005 